\title{
AlteraÇões ANATÔMICAS FoliaRes EM Eichhornia crassipes SubMetidas À APLICAÇÃo de Herbicidas ${ }^{1}$
}

\author{
Anatomical Leaf Changes in Eichhornia crassipes due to Herbicides Application
}

\author{
COSTA, N.V. ${ }^{2}$, MARTINS, D. ${ }^{3}$, RODELLA, R.A. ${ }^{4}$ e RODRIGUES-COSTA, A.C.P. ${ }^{2}$
}

\begin{abstract}
RESUMO - O presente trabalho teve por objetivo avaliar a eficiência do controle dos herbicidas 2,4-D, diquat e glyphosate e as alterações anatômicas do limbo foliar provocadas pelos produtos em plantas de Eichhornia crassipes, coletadas nos reservatórios do complexo CESP. As plantas foram cultivadas em caixas-d'água sob condições de campo e, quando atingiram estádio de pleno desenvolvimento vegetativo, foram pulverizadas com soluções de diquat a $400 \mathrm{~g}$ i.a.ha-1, 2,4-D a $1.340 \mathrm{~g}$ e.a. ha ${ }^{-1}$ e glyphosate a $4.320 \mathrm{~g}$ e.a. ha-1 em associação com o adjuvante Silwet L-77 a 0,01\% v v ${ }^{-1}$. Foi utilizada uma testemunha sem aplicação de herbicida. A seguir, foram realizadas avaliações de controle e das seguintes características anatômicas quantitativas das regiões da nervura central e da internervural do limbo foliar: porcentagem da epiderme adaxial e abaxial, porcentagem da endoderme, porcentagem do feixe vascular, porcentagem de lacunas do aerênquima, porcentagem de parênquima e espessura foliar $(\mu \mathrm{m})$. Com base nos resultados, os principais caracteres anatômicos quantitativos das regiões da nervura central e internervural do limbo foliar que sofreram alterações após a aplicação dos herbicidas foram a porcentagem da epiderme adaxial, a porcentagem da endoderme e a espessura foliar. Os herbicidas diquat e 2,4-D foram os que mais promoveram alterações nos caracteres anatômicos quantitativos das regiões da nervura central e internervural do limbo foliar das plantas de E. crassipes. Apenas o glyphosate apresentou controle de $100 \%$ das plantas aos 22 dias após a aplicação, quando comparado com o observado na testemunha sem herbicida.
\end{abstract}

Palavras-chave: anatomia foliar, planta aquática, planta daninha, controle químico.

\begin{abstract}
The aim of this work was to evaluate the efficacy of 2,4-D, diquat and glyphosate control and the leafblade anatomic changes provided by these herbicides in Eichhornia crassipes, collected in the reservoirs of the CESP complex. The plants were grown in reservoirs containing water under field conditions. When these plants reached the stage of full vegetative development, they were sprayed with solutions of diquat at $400 \mathrm{~g}$ a.i. ha-1, 2, 4-D at 1.340 g a.e. ha-1 and glyphosate at $4.320 \mathrm{~g}$ a.e. ha ${ }^{1}$ in combination with Silwet $L-77$ at $0.01 \% v v^{-1}$. A control without herbicide was also used. Evaluations were carried out of the control and of the following anatomical quantitative characteristics of the vein and intervein central regions of the leaf blade: percentage of the adaxial and abaxial epidermis, percentage of the endoderm, percentage of vascular bundle, percentage of the aerenchyma lacuna, percentage of parenchyma and leafthickness $(\mu \mathrm{m})$. According to the results, the main anatomical quantitative characters of the vein and intervein central regions of the leaf blade that changed after herbicide application were: percentage of the adaxial epidermis, percentage of the endoderm, and leaf thickness. Diquat and 2,4-D promoted changes in the anatomical quantitative characters of the vein and intervein central regions of the leaf blade in $\boldsymbol{E}$. crassipes. Only glyphosate provided 100\% of $\boldsymbol{E}$. crassipes control at 22 days after application, compared with the control without herbicide.
\end{abstract}

Keywords: leaf anatomy, aquatic plant, weed, chemical control.

Recebido para publicação em 19.3.2010 e na forma revisada em 18.2.2011.

2 Professor Adjunto, Universidade Estadual do Oeste do Paraná - UNIOESTE/CCA, Marechal Cândido Rondon-PR, $<$ neumarciovc@hotmail.com>; ${ }^{3}$ Professor Adjunto, Dep. de Produção Vegetal, Faculdade de Ciências Agronômicas - FCA/UNESP, Fazenda Lageado, Caixa Postal 237, 18603-970 Botucatu-SP; ${ }^{4}$ Professor Adjunto, Dep. de Botânica, Instituto de Biociências de Botucatu - UNESP, Botucatu-SP. 


\section{INTRODUÇÃO}

A vegetação aquática apresenta importantes funções para a manutenção do equilíbrio do ambiente em que vive, como na proteção e estabilização das margens de rios, lagos e represas, diminuindo os efeitos erosivos da água; ela é fundamental também na cadeia alimentar, promovendo a oxigenação e a depuração da água. Entretanto, alterações no ambiente, seja pela mudança na qualidade da água e/ou pela construção de reservatórios, alterando os niveis hidrométricos do ecossistema, podem acarretar desenvolvimento indesejado das populações de plantas aquáticas e ocasionar prejuízos significativos ao ambiente, bem como ao uso múltiplo da água (Tanaka, 2001; Thomaz, 2002).

Do ponto de vista ambiental, algumas espécies de crescimento rápido podem suprimir outras menos agressivas, desejáveis para manutenção da diversidade, ou modificar negativamente algumas características físicas da água (Cardoso et al., 2002). O lançamento de efluentes de origem urbana e industrial, além do aporte de fertilizantes utilizados na agricultura para os corpos hídricos, podem ser citados como outras possiveis causas que estimulam a proliferação desordenada das espécies de plantas aquáticas.

Os métodos de controle de plantas aquáticas mais utilizados normalmente são controle biológico, químico e mecânico. Entre estes, destaca-se o controle químico como a alternativa mais econômica, além de ser eficiente. Vários trabalhos realizados por diferentes autores têm comprovado a eficiência do controle químico e a sua segurança sobre os organismos aquáticos através do uso de diversos herbicidas (Murphy \& Barrett, 1990; Martins et al., 1999; Hofstra et al., 2001; Parsons et al., 2001; Martins et al., 2002; Neves et al., 2002).

Entretanto, a morfologia das plantas, principalmente das folhas, influencia na quantidade do herbicida interceptado e retido. Contudo, é a anatomia das folhas que praticamente determina a facilidade com que esses produtos serão absorvidos (Procópio et al., 2003). Segundo Ferreira et al. (2002a,b) e Procópio et al. (2003), o estudo anatômico de folhas pode melhorar o entendimento sobre as barreiras que cada espécie impõe à absorção dos herbicidas e, assim, fornecer subsídios para a busca de estratégias que superem esses obstáculos.

Dessa forma, a análise anatômica foliar pode ser aplicada com sucesso na identificação de espécies suscetiveis, tolerantes ou resistentes a determinado produto químico, bem como na descrição dos sintomas fitotóxicos, contribuindo com os estudos de seletividade de herbicidas a espécies terrestres ou aquáticas.

Dentre as várias espécies que proliferam nos reservatórios das usinas hidrelétricas do Estado de São Paulo, destaca-se em importância Eichhornia crassipes, que, de acordo com Kissmann \& Groth (1997), é uma planta aquática flutuante, podendo apresentar enraizamento se o lençol de água for pouco profundo; a reprodução principal é vegetativa, a partir de talos, nos quais ocorre brotamento. No entanto, apesar da importância que representam os recursos hídricos para o Brasil na produção de energia elétrica, na atividade pesqueira e turismo, por exemplo, existem poucas informações na literatura sobre as comunidades vegetais presentes nos reservatórios, bem como sobre a eficiência dos herbicidas no controle das espécies de plantas aquáticas.

O presente trabalho teve por objetivo avaliar os herbicidas 2,4-D, diquat e glyphosate no controle de plantas de E. crassipes e as prováveis alterações anatômicas do limbo foliar proporcionadas pelos produtos.

\section{MATERIAL E MÉTODOS}

O experimento foi conduzido no Núcleo de Pesquisas Avançadas em Matologia - NUPAM, do Departamento de Produção Vegetal, pertencente à Faculdade de Ciências Agronômicas de Botucatu/UNESP, e no Departamento de Botânica do Instituto de Biociências de Botucatu/UNESP. O local do experimento apresenta as seguintes coordenadas geográficas: latitude de $22^{\circ} 07^{\prime} 56^{\prime}$ ' S, longitute de $74^{\circ} 66^{\prime} 84^{\prime \prime} \mathrm{W}$ Gr. e altitude de $762 \mathrm{~m}$.

Foram avaliadas plantas de E. crassipes coletadas nos reservatórios do complexo CESP do Estado de São Paulo e reproduzidas por meio de suas estruturas de dispersão vegetativa, 
acondicionadas em pleno sol, em caixas-d'água de fibra de vidro de $350 \mathrm{~L}$, contendo uma camada de $30 \mathrm{~cm}$ de solo no fundo. Os herbicidas foram aplicados em pós-emergência, quando as plantas atingiram pleno desenvolvimento vegetativo (antes do florescimento), utilizandose um pulverizador costal, pressurizado por $\mathrm{CO}_{2}$ e equipado com um reservatório de $2 \mathrm{~L}$.

O equipamento foi regulado para proporcionar um consumo de calda de 200 ha ${ }^{1}$. A barra de aplicação foi equipada com duas pontas tipo jato plano Teejet XR 110.02, distanciadas $50 \mathrm{~cm}$ entre si. Os tratamentos utilizados foram os herbicidas diquat (400 g i.a. ha ${ }^{-1}$ do produto comercial Reward), 2,4-D (1.340 g e.a ha ${ }^{-1}$ do produto comercial DMA 806 BR) e glyphosate (4.320 g e.a. ha ${ }^{-1}$ do produto comercial Rodeo) com a adição do adjuvante Silwet L-77, na concentração do produto comercial de $0,01 \% \mathrm{v} \mathrm{v}^{-1}$, além de uma testemunha sem aplicação. Foram realizadas avaliações de controle após 1, 5, 10, 16, 22 e 162 dias após a aplicação (DAA) dos produtos.

As avaliações de controle foram visuais, utilizando-se escala percentual de notas, em que 0 (zero) corresponde a nenhuma injúria demonstrada pela planta e 100 (cem) à morte das plantas, de acordo com a metodologia proposta pela Sociedade Brasileira da Ciência das Plantas Daninhas - SBCPD (1995). Os parâmetros utilizados para o estabelecimento das notas foram: quantidade de plantas mortas, inibição do crescimento, quantidade e uniformidade das injúrias e capacidade de rebrota das plantas.

Quanto aos estudos anatômicos, nas datas de 1 e 16 DAA, foram coletadas porções da região mediana do limbo foliar das plantas que apresentaram sintomas externos de intoxicação e da testemunha. Foram tomadas as porções do terço médio do limbo foliar, compreendendo as regiões da nervura central e da internervural (situada entre a nervura central e o bordo do limbo foliar), fixando-as em FAA 50 (formaldeído + ácido acético glacial + álcool 50\%) durante 48 horas, sendo, posteriormente, conservadas em álcool 70\% (Johansen, 1940), utilizando-se quatro repetições.

As amostras do material foliar foram desidratadas em série etílica ascendente e, em seguida, infiltradas em resina glicolmetacrilato, seguindo-se a técnica de Gerrits (1991). Realizaram-se cortes transversais com espessura de 8 a $10 \mu \mathrm{m}$, utilizando micrótomo rotatório; os cortes foram submetidos à coloração com azul de toluidina 0,05\% (O'Brien et al., 1964) e montados em resina sintética.

Para a quantificação das estruturas anatômicas das regiões da nervura central e da internervural do limbo foliar, os limites e os contornos dos tecidos foram desenhados com o auxílio de microscópio de projeção, e as mensurações, feitas com auxílio de mesa digitalizadora acoplada a um computador equipado com o programa Sistema de Planimetria (SPLAN), desenvolvido pelo CINAG - UNESP.

Foram avaliadas as seguintes características anatômicas quantitativas das regiões da nervura central e da internervural do limbo foliar: porcentagem da epiderme adaxial e abaxial, porcentagem da endoderme, porcentagem do feixe vascular, porcentagem de lacunas do aerênquima, porcentagem de parênquima e espessura foliar $(\mu \mathrm{m})$.

Os tratamentos foram dispostos em delineamento experimental inteiramente casualizado; para as avaliações das alterações anatômicas do limbo foliar foi adotado esquema fatorial $4 \times 2$ (quatro herbicidas $\mathrm{x}$ duas épocas de avaliações), com quatro repetições. Os dados de controle e das alterações anatômicas do limbo foliar foram analisados estatisticamente, empregando-se o teste LSD a 5\% de probabilidade; os dados originais, expressos em porcentagem, foram transformados utilizando-se arco seno $\sqrt{ } \mathrm{x} / 100$ (Pimentel-Gomes, 1976).

\section{RESULTADOS E DISCUSSÃO}

A porcentagem de controle proporcionada pelos herbicidas diquat, 2,4-D e glyphosate em plantas de E. crassipes está apresentada na Tabela 1. Verificou-se que a partir de 1 DAA o herbicida diquat promoveu $60 \%$ de controle das plantas em relação a testemunha. Aos 10 DAA, todos os herbicidas apresentaram controle acima de $90 \%$, e somente o glyphosate mostrou controle de $100 \%$ das plantas aos 22 DAA. É importante ressaltar que a partir de 162 DAA as plantas dos tratamentos com os herbicidas diquat e 2,4-D apresentaram rebrota, reduzindo o controle a 15 e $30 \%$, respectivamente. 
Assim, esse fato evidencia que, apesar da elevada eficiência desses herbicidas no controle de E. crassipes, torna-se importante a realização de monitoramento constante nas áreas aplicadas, para que se possa avaliar a necessidade de uma reaplicação e evitar a reinfestação do local.

Contudo, esses resultados corroboram os obtidos por Martins et al. (2002) e Neves et al. (2002), que avaliaram os efeitos dos herbicidas diquat, 2,4-D e glyphosate no controle do aguapé (E. crassipes) e obtiveram resultados excelentes e próximos a $100 \%$ de eficiência, aos 20 dias após a aplicação dos produtos. Da mesma forma, Martins et al. (1999), avaliando os herbicidas 2,4-D (1.200 g e.a. ha $\left.{ }^{1}\right) \mathrm{e}$ glyphosate (3.360 g e.a. ha $\left.{ }^{-1}\right)$ no controle de E. crassipes, constataram, respectivamente, eficiência de 97,5 e 96\% aos 35 dias após a aplicação dos herbicidas.
Nas Tabela 2 estão apresentados os valores do teste $\mathrm{F}$ e do coeficiente de variação dos caracteres anatômicos quantitativos da região da nervura central e internervural do limbo foliar de plantas de E. crassipes.

Na Tabela 3 podem-se verificar os valores médios dos caracteres anatômicos quantitativos da região da nervura central do limbo foliar das plantas de E. crassipes. Observou-se que apenas os caracteres de porcentagem da endoderme e espessura foliar apresentaram interação entre os fatores herbicidas e a época de avaliação. O caráter porcentagem da epiderme adaxial apresentou diferença estatística somente para o fator herbicida (Tabela 2). Assim, ao analisar as médias obtidas pelos herbicidas diquat $(4,78 \%)$ e glyphosate $(4,72 \%)$ para o caráter porcentagem da epiderme adaxial, observou-se que foram inferiores, em relação à testemunha $(6,30 \%)$.

Tabela 1 - Porcentagem de controle das plantas de E. crassipes após a aplicação dos herbicidas. Botucatu-SP, 2005

\begin{tabular}{|c|c|c|c|c|c|c|c|}
\hline \multirow{2}{*}{ Tratamento } & \multirow{2}{*}{$\begin{array}{c}\text { Dose } \\
\text { (i.a. } \mathrm{g} \mathrm{ha}^{-1} \text { ) }\end{array}$} & \multicolumn{6}{|c|}{ Dias após a aplicação } \\
\hline & & 1 & 5 & 10 & 16 & 22 & 162 \\
\hline Testemunha & --- & $0,0 \mathrm{c}$ & $0,0 \mathrm{~d}$ & $0,0 \mathrm{c}$ & $0,0 \mathrm{~b}$ & $0,0 \mathrm{c}$ & $0,0 \mathrm{~b}$ \\
\hline Diquat & $400 *$ & $60,0 \mathrm{a}$ & $89,3 \mathrm{a}$ & $97,0 \mathrm{ab}$ & $97,5 \mathrm{a}$ & $94,8 \mathrm{~b}$ & $15,0 \mathrm{a}$ \\
\hline $2,4-\mathrm{D}$ & 1.340 & $7,5 \mathrm{~b}$ & $57,5 \mathrm{~b}$ & $95,0 \mathrm{~b}$ & $97,8 \mathrm{a}$ & $96,8 \mathrm{~b}$ & $30,0 \mathrm{a}$ \\
\hline Glyphosate + Silwet L-77 & $4.320 *+0,01 \mathrm{v} \mathrm{v}^{-1}$ & $0,0 \mathrm{c}$ & $47,5 \mathrm{c}$ & $98,0 \mathrm{a}$ & $99,0 \mathrm{a}$ & $100,0 \mathrm{a}$ & $0,0 \mathrm{~b}$ \\
\hline $\mathrm{F}_{\text {Tratamento }}$ & & $834,414 * *$ & $273,304 * *$ & $955,259^{* *}$ & $1.326,430^{* *}$ & $355,746^{* *}$ & $11,443^{* *}$ \\
\hline $\mathrm{CV}(\%)$ & & 9,96 & 8,79 & 4,32 & 3,66 & 7,12 & 72,28 \\
\hline d.m.s. & & 2,554 & 5,546 & 3,989 & 3,490 & 6,829 & 14,438 \\
\hline
\end{tabular}

Os dados originais de porcentagem foram transformados em arco seno $\sqrt{\mathrm{x}} / 100$. Médias seguidas de mesma letra, minúsculas na coluna, não diferem estatisticamente entre si a $5 \%$ de probabilidade pelo teste LSD. ${ }^{* *}$ significativo a $1 \%$ de probabilidade. ${ }^{*}$ equivalente ácido.

Tabela 2 - Valores do teste F e do coeficiente de variação dos caracteres anatômicos quantitativos da região da nervura central e internervural do limbo foliar das plantas de E. crassipes. Botucatu-SP, 2005

\begin{tabular}{|c|c|c|c|c|c|c|c|}
\hline Fonte de variação & $\begin{array}{l}\text { \% Epiderme } \\
\text { adaxial }\end{array}$ & $\begin{array}{l}\text { \% Epiderme } \\
\text { abaxial }\end{array}$ & $\%$ Endoderme & $\begin{array}{l}\% \text { Feixe } \\
\text { vascular }\end{array}$ & $\%$ Lacunas & \% Parênquima & $\begin{array}{r}\text { Espessura } \\
\text { foliar }(\mu \mathrm{m})\end{array}$ \\
\hline & \multicolumn{7}{|c|}{ Região da nervura central } \\
\hline Herbicidas $(\mathrm{H})$ & $3,559 *$ & $1,160^{\mathrm{ns}}$ & $10,931 * *$ & $1,351^{\mathrm{ns}}$ & $2,612^{\text {ns }}$ & $2,269^{\text {ns }}$ & $4,468^{*}$ \\
\hline Época de Avaliação (E) & $1,048^{\text {ns }}$ & $2,600^{\text {ns }}$ & $4,592 *$ & $4,056^{\mathrm{ns}}$ & $0,513^{\mathrm{ns}}$ & $2,925^{\mathrm{ns}}$ & $2,105^{\mathrm{ns}}$ \\
\hline$(\mathrm{H}) \times(\mathrm{E})$ & $0,242^{\text {ns }}$ & $1,329^{\text {ns }}$ & $4,435^{*}$ & $1,549^{\mathrm{ns}}$ & $0,200^{\text {ns }}$ & $0,406^{\mathrm{ns}}$ & $3,238^{*}$ \\
\hline \multirow[t]{2}{*}{ C.V. $(\%)$} & 11,81 & 13,91 & 10,37 & 10,62 & 20,71 & 10,33 & 23,24 \\
\hline & \multicolumn{7}{|c|}{ Região internervural } \\
\hline Herbicidas $(\mathrm{H})$ & $3,319 *$ & $1,997^{\mathrm{ns}}$ & $11,280 * *$ & $0,515^{\mathrm{ns}}$ & $1,410^{\mathrm{ns}}$ & $2,606^{\mathrm{ns}}$ & $5,735 * *$ \\
\hline Época de Avaliação (E) & $1,331^{\text {ns }}$ & $1,119^{\mathrm{ns}}$ & $0,467 * *$ & $0,467^{\text {ns }}$ & $2,583^{\mathrm{ns}}$ & $0,078^{\mathrm{ns}}$ & $7,966 * *$ \\
\hline$(\mathrm{H}) \times(\mathrm{E})$ & $1,39^{\text {ns }}$ & $1,410^{\text {ns }}$ & $2,068 * *$ & $2,068^{\mathrm{ns}}$ & $1,265^{\mathrm{ns}}$ & $0,548^{\mathrm{ns}}$ & $1,538^{\text {ns }}$ \\
\hline $\mathrm{CV}(\%)$ & 11,06 & 132,54 & 9,51 & 10,38 & 20,546 & 11,31 & 21,59 \\
\hline
\end{tabular}

** significativo a $1 \%$ de probabilidade, $*$ significativo a $5 \%$ de probabilidade, e ${ }^{\text {ns }}$ não significativo. 
Quanto à porcentagem da endoderme, constatou-se diferença entre as épocas de avaliações somente para o 2,4-D, que apresentou o menor valor aos 16 DAA em relação ao verificado na primeira época de avaliação. Da mesma maneira, o 2,4-D foi o único herbicida que promoveu redução deste caráter em relação à testemunha aos 16 DAA.

Com relação à espessura foliar, verificaram-se diferenças estatísticas para épocas de avaliação apenas para o herbicida 2,4-D, e o valor obtido aos 16 DAA foi inferior ao da primeira avaliação. Entretanto, na primeira época de avaliação os herbicidas não diferiram

Tabela 3 - Valores médios dos caracteres anatômicos quantitativos da região da nervura central do limbo foliar das plantas de E. crassipes. Botucatu-SP, 2005

\begin{tabular}{|c|c|c|c|c|}
\hline \multirow{2}{*}{$\mathrm{DAA}^{1 /}$} & \multicolumn{4}{|c|}{ Herbicida } \\
\hline & Testemunha & Diquat & 2,4-D & Glyphosate \\
\hline & \multicolumn{4}{|c|}{$\%$ Epiderme adaxial } \\
\hline 1 & 6,03 & 4,88 & 5,78 & 4,36 \\
\hline 16 & 6,58 & 4,67 & 6,56 & 5,08 \\
\hline \multirow[t]{2}{*}{ Média } & $6,30 \mathrm{a}$ & $4,78 \mathrm{~b}$ & $6,17 \mathrm{a}$ & $4,72 \mathrm{~b}$ \\
\hline & \multicolumn{4}{|c|}{$\%$ Epiderme abaxial } \\
\hline 1 & 3,98 & 4,00 & $\begin{array}{l}3,49 \\
\end{array}$ & 3,17 \\
\hline 16 & 4,84 & 3,41 & 4,94 & 3,87 \\
\hline \multirow[t]{2}{*}{ Média } & 4,41 & 3,70 & 4,22 & 3,52 \\
\hline & \multicolumn{4}{|c|}{$\%$ Endoderme } \\
\hline 1 & $20,22 \mathrm{aA}$ & $17,54 \mathrm{aA}$ & $15,48 \mathrm{aA}$ & $18,53 \mathrm{aA}$ \\
\hline 16 & $17,82 \mathrm{aA}$ & $19,95 \mathrm{aA}$ & $7,87 \mathrm{bB}$ & $17,39 \mathrm{aA}$ \\
\hline \multirow[t]{2}{*}{ Média } & 19,02 & 18,75 & 11,68 & 17,96 \\
\hline & \multicolumn{4}{|c|}{$\%$ Feixe vascular } \\
\hline 1 & 9,16 & 8,58 & 9,28 & 10,45 \\
\hline 16 & 9,05 & 8,71 & 6,36 & 8,38 \\
\hline \multirow[t]{2}{*}{ Média } & 9,11 & 8,65 & 7,82 & 9,41 \\
\hline & \multicolumn{4}{|c|}{ \% Lacunas do aerênquima } \\
\hline 1 & 18,35 & 23,31 & 22,81 & 26,52 \\
\hline 16 & 13,31 & 20,80 & 23,05 & 25,62 \\
\hline \multirow[t]{2}{*}{ Média } & 15,83 & 22,05 & 22,93 & 26,07 \\
\hline & \multicolumn{4}{|c|}{$\%$ Parênquima } \\
\hline 1 & 42,26 & 41,69 & 43,16 & 36,98 \\
\hline 16 & 48,40 & 42,46 & 51,22 & 39,67 \\
\hline \multirow[t]{2}{*}{ Média } & 45,33 & 42,07 & 47,19 & 38,32 \\
\hline & \multicolumn{4}{|c|}{ Espessura foliar $(\mu \mathrm{m})$} \\
\hline 1 & $1.085,30 \mathrm{abA}$ & $1.148,39 \mathrm{aA}$ & $1.051,42 \mathrm{abA}$ & $817,52 \mathrm{bA}$ \\
\hline 16 & $850,78 \mathrm{bcA}$ & $1.257,52 \mathrm{aA}$ & $587,00 \mathrm{cB}$ & $945,74 \mathrm{abA}$ \\
\hline Média & 968,04 & $1.202,95$ & 819,21 & 881,63 \\
\hline
\end{tabular}

Os dados originais de porcentagem foram transformados em arco seno $\sqrt{\mathrm{X}}_{\mathrm{X}} / 100$. Médias seguidas de mesma letra, minúsculas na linha e maiúsculas na coluna, não diferem estatisticamente entre si a 5\% de probabilidade pelo teste LSD

1/ DAA - dias após a aplicação. da testemunha, enquanto aos 16 DAA apenas o herbicida diquat apresentou valor superior ao da testemunha.

Na Tabela 4 podem-se observar os valores médios dos caracteres anatômicos quantitativos da região internervural do limbo foliar das plantas de E. crassipes. Verificou-se que os caracteres porcentagem de epiderme abaxial, feixe vascular, lacunas do aerênquima e parênquima não foram alterados com o uso dos herbicidas, ao passo que apenas o caráter porcentagem da endoderme apresentou interação entre os fatores herbicidas e época de avaliação (Tabela 2).

Tabela 4 - Valores médios dos caracteres anatômicos quantitativos da região internervural do limbo foliar das plantas de E. crassipes. Botucatu-SP, 2005

\begin{tabular}{|c|c|c|c|c|c|}
\hline \multirow[b]{2}{*}{$\mathrm{DAA}^{1 /}$} & \multicolumn{5}{|c|}{ Herbicida } \\
\hline & $\begin{array}{l}\text { Teste- } \\
\text { munha }\end{array}$ & Diquat & $2,4-\mathrm{D}$ & Glyphosate & Média \\
\hline & \multicolumn{5}{|c|}{$\%$ Epiderme adaxial } \\
\hline 1 & 7,55 & $7,3,38$ & 5,33 & 4,88 & 6,29 \\
\hline 16 & 5,81 & 6,46 & 4,86 & 5,80 & 5,73 \\
\hline \multirow{2}{*}{ Média } & $6,68 \mathrm{ab}$ & $6,92 \mathrm{~A}$ & $5,09 \mathrm{c}$ & $5,34 \mathrm{bc}$ & 6,01 \\
\hline & \multicolumn{5}{|c|}{$\%$ Epiderme abaxial } \\
\hline 1 & 5,10 & \begin{tabular}{|l|l|}
4,90 \\
\end{tabular} & 4,00 & 3,58 & 4,40 \\
\hline 16 & 3,48 & 4,94 & 3,41 & 4,10 & 3,98 \\
\hline \multirow[t]{2}{*}{ Média } & 4,29 & 4,92 & 3,71 & 3,84 & 4,19 \\
\hline & \multicolumn{5}{|c|}{$\%$ Endoderme } \\
\hline 1 & $21,54 \mathrm{aA}$ & $17,36 \mathrm{abA}$ & $16,87 \mathrm{bA}$ & $17,07 \mathrm{abA}$ & 18,21 \\
\hline 16 & $15,57 \mathrm{aB}$ & $6,51 \mathrm{bB}$ & $17,58 \mathrm{aA}$ & $18,65 \mathrm{aA}$ & 14,58 \\
\hline \multirow[t]{2}{*}{ Média } & 18,56 & 11,94 & 17,22 & 17,86 & 16,39 \\
\hline & \multicolumn{5}{|c|}{$\%$ Feixe vascular } \\
\hline 1 & 8,30 & 8,18 & 7,84 & 7,24 & 7,89 \\
\hline 16 & 7,48 & 6,35 & 7,46 & 9,06 & 7,59 \\
\hline \multirow[t]{2}{*}{ Média } & 7,89 & 7,27 & 7,65 & 8,15 & 7,74 \\
\hline & \multicolumn{5}{|c|}{ \% Lacunas do aerênquima } \\
\hline 1 & 17,93 & 13,85 & 22,94 & 27,52 & 20,56 \\
\hline 16 & 23,10 & 24,82 & 28,11 & 23,09 & 24,78 \\
\hline \multirow[t]{2}{*}{ Média } & 20,52 & 19,34 & 25,52 & 25,30 & 22,67 \\
\hline & \multicolumn{5}{|c|}{ \% Parênquima } \\
\hline 1 & 39,57 & 48,32 & 43,01 & 39,69 & 42,65 \\
\hline 16 & 44,57 & 50,92 & 38,59 & 39,31 & 43,35 \\
\hline \multirow[t]{2}{*}{ Média } & 42,07 & 49,62 & 40,80 & 39,50 & 43,00 \\
\hline & \multicolumn{5}{|c|}{ Espessura foliar $(\mu \mathrm{m})$} \\
\hline 1 & 492,19 & 398,22 & 522,79 & 598,92 & $503,03 \mathrm{~A}$ \\
\hline 16 & 521,30 & 276,43 & 383,28 & 439,83 & $405,21 \mathrm{~B}$ \\
\hline Média & $506,74 \mathrm{a}$ & $337,33 \mathrm{~B}$ & $453,03 \mathrm{a}$ & $519,38 \mathrm{a}$ & \\
\hline
\end{tabular}

Os dados originais de porcentagem foram transformados em arco seno $\sqrt{ } \mathrm{x} / 100$. Médias seguidas de mesma letra, minúsculas na linha e maiúsculas na coluna, não diferem estatisticamente entre si a $5 \%$ de probabilidade pelo teste LSD

1/ DAA - dias após a aplicação. 
Para a porcentagem de epiderme adaxial, somente o herbicida 2,4-D promoveu alteração deste caráter, apresentando valor médio inferior ao da testemunha. Contudo, as plantas de $E$. crassipes apresentaram espessura foliar reduzida quando se utilizou o herbicida diquat, bem como verificou-se que aos 16 DAA a média obtida foi menor em relação à primeira época de avaliação .

$\mathrm{Na}$ interação entre os fatores herbicidas e época de avaliação para a porcentagem da endoderme, verificaram-se diferenças estatísticas nas épocas de avaliação para o herbicida diquat, sendo o valor obtido aos 16 DAA inferior ao da primeira avaliação, porém esse resultado também foi verificado na testemunha. Entretanto, na primeira época de avaliação, o herbicida 2,4-D diferiu da testemunha, e aos 16 DAA apenas o herbicida diquat apresentou valor inferior ao da testemunha.

Segundo Murphy \& Barrett (1990) e Rodrigues \& Almeida (1998), o herbicida 2,4-D é idêntico às auxinas (AIA) e provoca intensa divisão celular no câmbio, endoderme, periciclo e floema; o encurtamento do tecido internervural das folhas e a epinastia são os sintomas mais evidentes nas dicotiledôneas. Esse fato pode explicar as alterações observadas nas regiões da nervura central e internervural do limbo foliar das plantas de E. crassipes. Contudo, no presente trabalho observou-se que, mesmo com controle superior a 90\% aos, 22 DAA, algumas plantas apresentaram inicio de rebrota. Esse fato pode ser justificado, provavelmente, pela grande variabilidade genética existente entre as plantas de E. crassipes, sendo possivel que algumas delas apresentem a capacidade de metabolizar o 2,4-D após um período longo de tempo e se regenerar.

Da mesma maneira, apesar de o diquat ter apresentado eficiência de controle superior a 95\% aos 22 DAA, algumas plantas apresentaram início de rebrota a partir desse período. No entanto, isso pode ser justificado pelo fato de o diquat ser um herbicida de contato e possuir baixa translocação na planta. Assim, no momento da pulverização, em algumas regiões da planta pode não ter ocorrido deposição do produto, sendo preservados caracteres anatômicos capazes de regenerar a planta.
O glyphosate, herbicida sistêmico que atua sobre a atividade enzimática responsável pela formação dos aminoácidos triptofano, tirosina e fenilalanina e outros produtos endógenos, apresentou alta eficácia no controle de plantas de E. crassipes.

Com base nos resultados deste trabalho, ressalta-se que os principais caracteres anatômicos quantitativos das regiões da nervura central e internervural do limbo foliar que sofreram alterações após a aplicação dos herbicidas foram: porcentagem da epiderme adaxial, porcentagem da endoderme e espessura foliar; as alterações mais evidentes foram observadas na segunda época de avaliação (16 DAA), quando os herbicidas apresentaram controle das plantas de E. crassipes superior a $95 \%$.

Os herbicidas diquat e 2,4-D promoveram o maior número de alterações nos caracteres anatômicos quantitativos das regiões da nervura central e internervural do limbo foliar das plantas de E. crassipes. Entretanto, aos 162 DAA esses herbicidas permitiram rebrota das plantas, fato que não ocorreu quando se utilizou o glyphosate. Esses resultados evidenciam que o manejo químico de plantas de E. crassipes requer mais estudos de longo prazo, uma vez que essa espécie apresenta alta capacidade de regeneração e reprodução após a aplicação de herbicidas considerados eficientes, como o diquat e o 2,4-D.

\section{LITERATURA CITADA}

CARDOSO, L. R. et al. Variabilidade genética de acessos de aguapé coletados no Estado de São Paulo. Planta Daninha, v. 20, n. 1, p. 1-5, 2002. Edição Especial.

FERREIRA, E. A. et al. Estudos anatômicos de folhas de plantas daninhas. I. Nicandra physaloides, Solanum americanum e Raphanus raphanistrum. Planta Daninha, v. 20 , n. 2 , p. $159-167,2002 \mathrm{a}$.

FERREIRA, E. A. et al. Estudos anatômicos de folhas de plantas daninhas. II. Bidens pilosa, Emilia sonchifolia, Agerantum conyzoides e Sonchus asper. Planta Daninha, v. 20, n. 3 , p. $327-335,2002$ b.

GERRITS, P. O. The application of glycol metacrylate in histotechnology: same fundamental principles. Germany: Lica Gmgh, 1991. 80 p. 
HOFSTRA, D. E.; CLAYTON, J. S.; GETSINGER, K. D. Evaluation of selected herbicides for the control of exotic submerged weeds in New Zealand: II. The effects of turbidity on diquat and endothall efficacy. J. Aquat. Plant Manag. v. 39 , n. 1 , p. $25-27,2001$.

JOHANSEN, D. A. Plant microtechnique. New York: McGrow-Hill Book, 1940. 523 p.

KISSMANN, K. G.; GROTH, D. Plantas infestantes e nocivas. 2.ed. São Paulo: BASF, 1997. Tomo I. 825 p.

MARTINS, D. et al. Controle químico de plantas daninhas aquáticas em condições controladas-caixas d'água

Planta Daninha, v. 17, n. 2, p. 289-296, 1999.

MARTINS, D. et al. Controle químico de Pistia stratiotes, Eichhornia crassipes e Salvinia molesta em caixas d'água. Planta Daninha, v. 20, n. 1, p. 83-88, 2002. Edição Especial.

MURPHY, K. J.; BARRETT, P. R. F. Chemical control of aquatic weeds. In: PIETERSE, A. H.; MURPHY, K. J. Aquatic weeds: the ecology and management of nuisance aquatic vegetation. Oxford: Oxford University Press, 1990. p. 136-173

NEVES, T.; FOLONI, L. L.; PITELLI, R. A. Controle químico do aguapé (Eichhornia crassipes). Planta Daninha, v. 20 , n. 1, p. 89-97, 2002. Edição Especial.

O'BRIEN, T. P.; FEDER, N.; McCULLY, M. E. Polychmofic staing of plant cellular wall by toluidine blue. Protoplasma, v. 59 , p. $368-373,1964$.
PARSONS, J. K. et al. The use 2,4-D for selective control of an early infestation of eurasian watermilfoil in loon lake, Washington. J. Aquat. Plant Manag., v. 39, n. 2, p. 117-125, 2001.

PIMENTEL-GOMES, F. Curso de estatística experimental. 6.ed. Piracicaba: Binetti, 1976. 430 p

PROCÓPIO, S. O. et al. Estudos anatômicos de folhas de plantas daninhas de grande ocorrência no Brasil. III.

Galinsoga parviflora, Crotalaria incana, Conyza bonariensis e Ipomoea cairica. Planta Daninha, v. 21, n. 1, p. 1-9, 2003

RODRIGUES, B. N.; ALMEIDA, F. S. Guia de herbicidas. 4.ed. Londrina: 1998. 648 p.

SOCIEDADE BRASILEIRA DA CIÊNCIA DAS PLANTAS DANINHAS - SBCPD. Procedimentos para instalação, avaliação e análise de experimentos com herbicidas. Londrina: 1995.42 p.

TANAKA, R. H. Eficácia de fluoridone para o controle de Egeria ssp. em caixas d'água e em represa de pequeno porte sem fluxo de água. 2001. 157 f. Dissertação (Mestrado em Ciências Agronômicas) - Faculdade de Ciências Agronômicas, Universidade Estadual de Paulista, Botucatu, 2001 .

THOMAZ, S. M. Fatores ecológicos associados à colonização e ao desenvolvimento de macrófitas aquáticas e desafios de manejo. Planta Daninha, v. 20, n. 1, p. 21-33, 2002. Edição Especial. 